\title{
Fiber Laser Incorporating an Intracavity Microchannel for Refractive Index and Temperature Sensing
}

\author{
Chengbo Mou, Kaiming Zhou, Edward Davies, Lin Zhang, and Ian Bennion, Member, IEEE
}

\begin{abstract}
In this letter, we present a standard linear cavity fiber laser incorporating a microchannel for refractive index (RI) and temperature sensing. The microchannel of $\sim 6-\mu \mathrm{m}$ width created by femtosecond laser aided chemical etching provides an access to the external liquid; therefore, the laser cavity loss changes with the liquids of different RIs. Thus, at a fixed pump power, the output laser power will vary with the change of RI in the microchannel. The results show that the proposed sensing system has a linear response to both the surrounding medium RI and temperature. The RI sensitivity of the laser system is on the order of $10^{-5}$, while the temperature sensitivity is about $0.02{ }^{\circ} \mathrm{C}$. Both sensitivities could be further enhanced by employing a more sensitive photodetector and using higher pump power.
\end{abstract}

Index Terms-Femtosecond laser, fiber laser sensor, microchannel, refractive index (RI) sensor.

\section{INTRODUCTION}

$\mathbf{O}$ PTICAL fiber based sensors have been extensively studied during the past few decades due to their merits over their electronic counterparts. Recently, optical fiber sensors for refractive index (RI) sensing or biochemical sensing have drawn more attention. Ultraviolet inscribed in-fiber Bragg gratings (FBGs) that are widely employed as strain and temperature sensors can be modified to suit for RI sensing [1]-[3]. In these modified grating devices, the fiber cladding is removed completely through chemical etching which seriously alleviates the device strength. Alternatively, cladding mode coupling devices such as long-period fiber gratings [4] and excessively tilted fiber gratings [5] can provide intrinsically high sensitivity to external RI without modifying structure to degrade the device strength.

Recently, femtosecond (fs) laser inscription and machining technologies have been developed for wide use of microstructuring and deemed as one of the most effective approaches to inscribing microstructures onto silica materials and optical fibers, owing to its high peak power and spatial resolution. It has also been reported that glass fiber regions modified by fs laser have a much higher etching rate than the pristine material [6]. Very recently, the combined fs patterning and chemical etching approach has been adopted to fabricate microchannel in conventional optical fiber for RI sensing [7]. However, all these techniques are passive-device-based sensing schemes. They all employ an external broadband light source and optical spectrum

Manuscript received May 27, 2009; revised August 10, 2009. First published August 25, 2009; current version published October 02, 2009.

The authors are with the Photonics Research Group, School of Engineering and Applied Science, Aston University, Birmingham, B4 7ET, U.K. (e-mail: mouc@aston.ac.uk).

Color versions of one or more of the figures in this letter are available online at http://ieeexplore.ieee.org.

Digital Object Identifier 10.1109/LPT.2009.2030337 analyzer to demodulate the optical signal which significantly increases the cost of the sensor system. In addition, the fabrication of fiber-grating-based sensors require expensive phase mask and hydrogen loading that all add additional cost.

We report here a novel laser sensor system incorporating a microchannel in the cavity created by fs-laser aided chemical etching for RI and temperature sensing. This system involves only a basic Er-Yb fiber linear cavity pumped by a low-cost laser diode and a low-cost photodiode detector for signal demodulation. The proposed active sensor system has shown an RI sensitivity in the order of $10^{-5}$ which can be further increased by using a more sensitive photodetector. The ultrasmall volume of the microchannel provides access to the sensing liquid without degrading mechanical strength of the cavity. This new scheme represents an ideal option for in-fiber biochemical sensor solution for a wide range of potential applications.

\section{DEVICE PREPARATION AND LASER SySTEM CONFIGURATION}

\section{A. Microchannel Fabrication by fs-Laser Inscription Aided Chemical Etching}

The fabrication process of the microchannel consists of two major steps:

1) patterning the microchannel in the core of a Corning SMF-28 fiber sample;

2) etching the fs-laser inscribed fiber in hydrofluoric acid (HF) of 5\% concentration solution for selectively removal of the fs-laser patterned area.

The fs laser used for the microchannel inscription process is Ti : sapphire mode locked solid-state laser (from SpectraPhyics) which gives laser pulses centered at $800 \mathrm{~nm}$. The output pulses were then tightly focused onto the fiber using a $\times 100$ objective lens (with an numerical aperture of 0.55 and a working distance of $13 \mathrm{~mm}$ ). The pulsewidth of fs laser was measured to be $\sim 150$ fs with a repetition rate $\sim 1 \mathrm{kHz}$. The average pulse energy was measured to be $100 \mathrm{~nJ}$. The fiber was mounted on a 3-D air-bearing translation stage, and the microchannel was written by moving the stage along the cross section of the fiber. Following the fs-laser inscription, the fiber with designed microchannel was chemically etched in the HF solution for only $\sim 8 \mathrm{~min}$. The selective etching behavior helps with creating the microchannel as well as maintaining the structural integrity near the microchannel region. An ultrasonic bath was employed to enhance the penetration of HF solution into the fs-laser modified area. After the etching, a microscope system (Zeis Axioskop 2 mot plus) was used to inspect the microchannel structure. Fig. 1 shows the microscopy images of the created microchannel in the fiber core under a $20 \times$ oil immersion microscopic lens. The channel width of about $6 \mu \mathrm{m}$ was measured, which agrees well with the design parameter. 


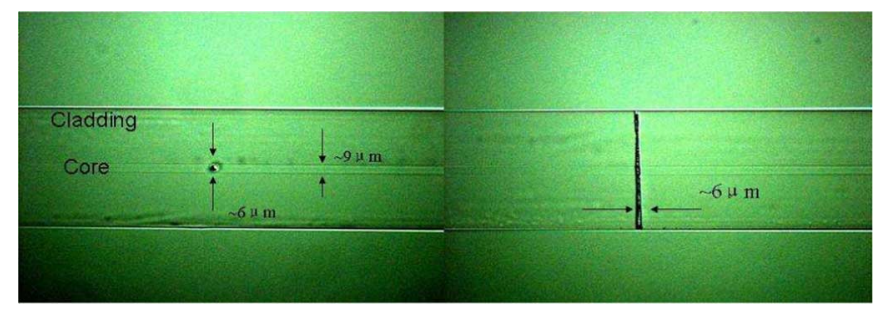

Fig. 1. Microscope images of a $\sim 6-\mu \mathrm{m}$ channel induced by fs-laser aided chemical etching in conventional SMF-28 fiber, examined by a $20 \times$ oil immersion microscopic lens.

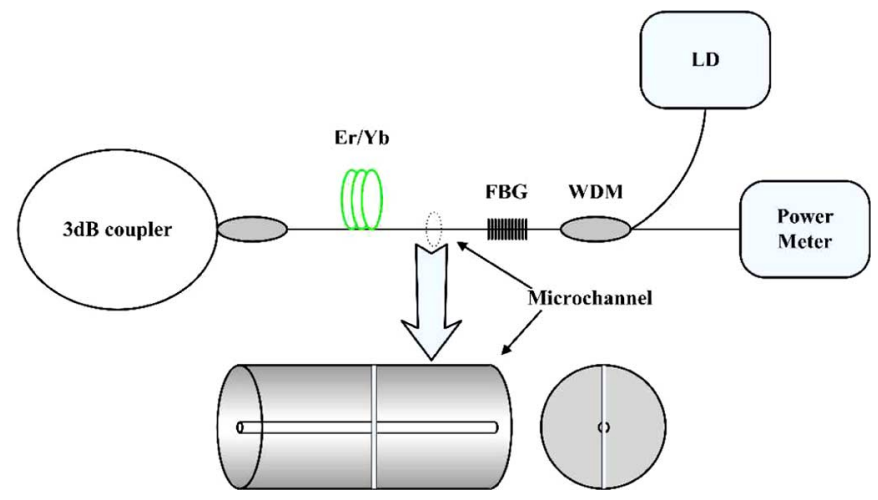

Fig. 2. Schematic diagram of proposed laser sensor system incorporating a microchannelled fiber.

\section{B. Laser System Configuration}

The experimental setup of the fiber laser is shown in Fig. 2. A standard linear cavity structure is employed. It consists of a $\sim 1$-m Er-Yb codoped fiber and a uniform FBG which has a reflectivity of $4.3 \mathrm{~dB}$ at $1551.4 \mathrm{~nm}$ functioning as a cavity mirror and output coupler of the fiber laser. A Sagnac loop formed by a $3-\mathrm{dB}$ coupler providing $\sim 90 \%$ reflection acts as the other high reflection mirror of the laser cavity. The laser is pumped through $980 / 1550$ wavelength-division multiplexing by a high-power 975-nm laser diode (Lumics) which can provide up to 500-mW pump power. A set of commercial laser diode driver (Newport 505B) and temperature controller (Newport 300) was employed to provide stable operation of the pump laser. A 40-cm SMF-28 fiber with the microchannel was spliced into the cavity placed between the $\mathrm{Er}-\mathrm{Yb}$ fiber and the FBG.

\section{SEnsing PRINCIPLE AND EXPERIMENTAL REsults}

\section{A. Sensing Principle}

As is well known, in a laser system, the round-trip gain suppresses any intracavity loss and amplifies the stimulated emission to give out coherence laser output. Therefore, the laser output power will change with any loss induced in the cavity while the other parameters kept the same such as pump power, gain medium, etc.

When the microchannel immerged with RI liquid, because the discontinuity in RI in this microchannel region, the cavity loss will be modified and the laser output will change accordingly. Thus, the correlation between the laser output power and RI provides a mechanism for RI sensing. In addition, the RI in the microchannel will change with temperature, thus the system can be used to measure temperature as well.
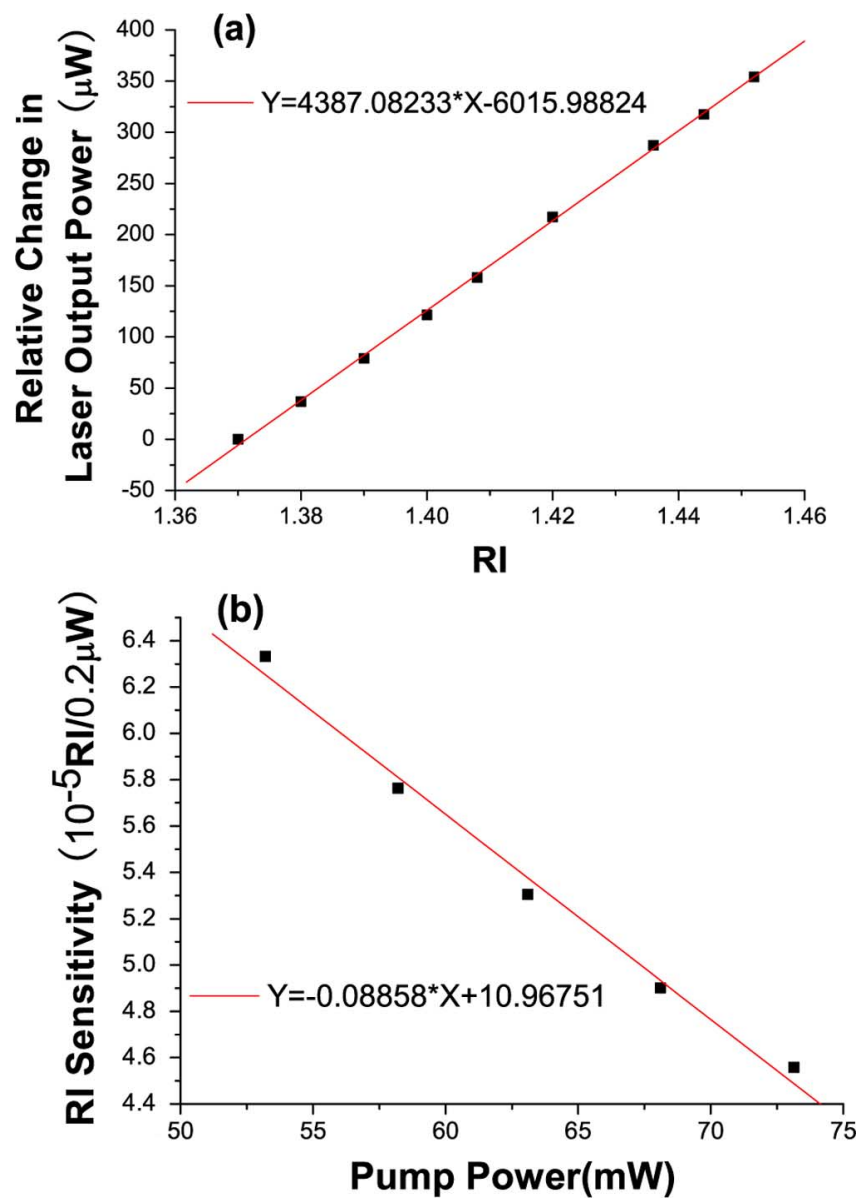

Fig. 3. (a) Relative change in fiber laser output power against the RI change when the pump power is fixed at $73.14 \mathrm{~mW}$. (b) RI sensitivity at various pump levels.

\section{B. RI and Temperature Measurements}

To investigate the RI response, we first fixed the diode pump power and examined the laser output while we applied a series of RI gels to the microchannel. The laser output was monitored by a high-speed power meter with a resolution of $0.2 \mu \mathrm{W}$ (EXFO PM1600 series). The segment of fiber containing the microchannel was fixed straight on a v-grooved metal plate in order to eliminate other effects such as temperature and bending. A series of certified RI gels (from Cargille Laboratories Inc.), which have values quoted with an accuracy of \pm 0.0002 , were used as the sensing liquids. After the measurement of each index gel, the microchannel part was cleaned by using acetone, methanol, and deionized water until the laser output power recovered to its original value when the microchannel was exposed to air. This was to make sure that in the microchannel area there was no residual index gel left.

The typical relative change in laser output power against RI is plotted in Fig. 3(a) with a fixed pump power of $73.14 \mathrm{~mW}$. The RI response shows a very good linearity from 1.37 to 1.452 . The calculated corresponding sensitivity in this region is $(4.5 \times$ $\left.10^{-5}\right) \mathrm{RI} / 0.2 \mu \mathrm{W}$. The pump power dependency of the RI sensitivity was also investigated and the correlation was plotted in Fig. 3(b). We can see from Fig. 3(b) that the RI sensitivity increases with increasing pump power.

We have also characterized the temperature response of the proposed laser sensor system containing a microchannel cavity. 


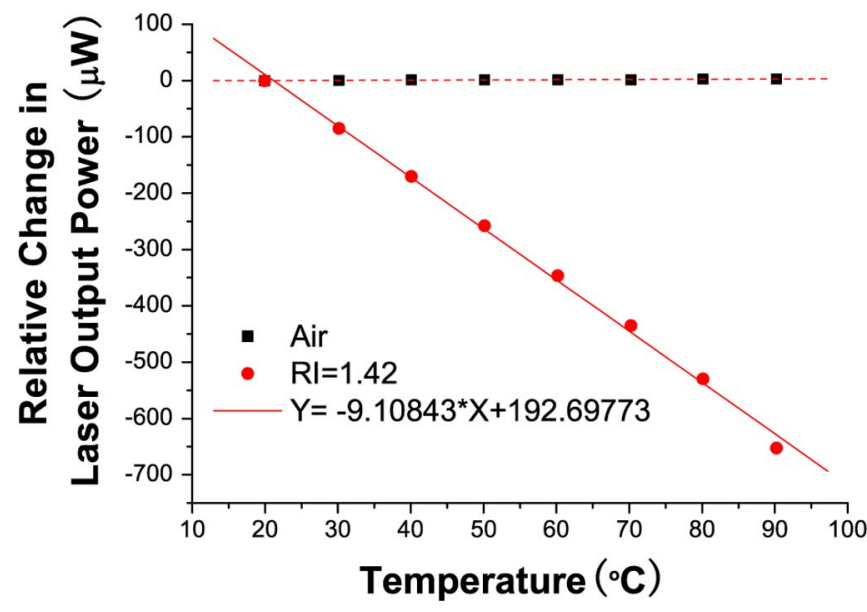

Fig. 4. Relative change in the laser output power $(\mathbf{\square})$ the microchannel is exposed to air; $(\bullet)$ the microchannel is immersed with liquid which has RI of 1.42 .

The microchannel part was mounted on a heating plate controlled by a commercial temperature controller (Lightwave LTD 5910B). We first measured the temperature response while the microchannel was exposed to air. As shown in Fig. 4, the laser output power has hardly changed (dashed horizontal line). This is because temperature increase does not affect the RI of air significantly.

The microchannel was then filled in with the index gel of $\mathrm{RI}=1.42$ and heated up from $20^{\circ} \mathrm{C}$ to $90^{\circ} \mathrm{C}$ with an increment of $10{ }^{\circ} \mathrm{C}$. The measured temperature-dependent laser output is plotted (solid line) in Fig. 4. We can see the laser output has a marked temperature responsivity: the output decreases with increasing temperature. This is due to the negative thermal coefficient of the index gel. From Fig. 4, we can estimate the temperature-induced laser power drop rate is about $-9.1 \mu \mathrm{W} /{ }^{\circ} \mathrm{C}$, which indicates that the system has a nominal temperature sensitivity of $-0.02{ }^{\circ} \mathrm{C} / 0.2 \mu \mathrm{W}$. The thermal responsivity characteristic makes this simple laser system suitable for temperature sensing; however, this may impose a temperature cross-sensitivity when the system is used for RI sensing. This effect could be overcome by keeping the microchannel part at constant temperature condition.

\section{DisCUSSION AND CONCLUSION}

It is obvious that the proposed fiber laser is not operating in a single longitudinal mode (SLM) regime. The multimode operation may induce mode competition, causing fluctuation in optical power detection. Thus, a stable SLM fiber laser is preferred more to increase the accuracy of the detected optical power.
We noticed that this sensing system does not respond to RI lower than 1.37. Because the sensing principle is based on RI-induced loss in the laser cavity, if the loss is too high, the lasing action will be suppressed. From the view of index continuity, if the RI in the microchannel is much less than that of the core, the scattering loss will be large. Apart from the scattering loss, the RI liquid may also induce absorption loss. Thus, when the filled liquid RI is close to 1.37 , the combined loss could be too large to get lasing.

It should be pointed out that the sensitivity of the RI depends on the resolution of the photodetector; therefore, even a smaller change of RI may be detected by using detectors with high sensitivity and low noise. Also, the RI measurement range can be simply increased by using a microchannel with smaller size. Furthermore, if higher pump power and longer gain medium are employed, more loss can be compensated, further increasing both sensing range and sensitivity.

In summary, we have presented an active RI and temperature sensing system based on a low-cost fiber laser detection system. The proposed laser sensor system shows high RI and temperature sensitivities as well as better mechanical sustainability compared to other chemically etched fiber-grating-based passive devices.

\section{REFERENCES}

[1] A. Iadicicco, A. Cusano, A. Cutolo, R. Bernini, and M. Giordano, "Thinned fiber Bragg gratings as high sensitivity refractive index sensors," IEEE Photon. Technol. Lett., vol. 16, no. 4, pp. 1149-1151, Apr. 2004.

[2] A. Iadicicco, S. Campopiano, A. Cutolo, M. Giordano, and A. Cusano, "Refractive index sensor based on microstructured fiber Bragg grating," IEEE Photon. Technol. Lett., vol. 17, no. 6, pp. 1250-1252, Jun. 2005.

[3] A. Iadicicco, S. Campopiano, A. Cutolo, M. Giordano, and A. Cusano, "Self temperature referenced refractive index sensor by non-uniform thinned fiber Bragg gratings," Sens. Actuators B, vol. 120, pp. 231-237, 2006.

[4] X. Shu, L. Zhang, and I. Bennion, "Sensitivity characteristics of longperiod fiber gratings," J. Lightw. Technol., vol. 20, no. 2, pp. 255-266, Feb. 2002.

[5] K. Zhou, L. Zhang, X. Chen, and I. Bennion, "Optic sensors of high refractive-index responsivity and low thermal cross sensitivity that use fiber Bragg gratings of $>80^{\circ}$ tilted structures," Opt. Lett., vol. 31, no. 9, pp. 1193-1195, 2006.

[6] C. Hnatovsky, R. S. Taylor, E. Simova, V. R. Bhardwaj, D. M. Rayner, and P. B. Corkum, "Polarization-selective etching in femtosecond laser-assisted microfluidic channel fabrication in fused silica," Opt. Lett., vol. 30, no. 14, pp. 1867-1869, 2005.

[7] Y. Lai, K. Zhou, L. Zhang, and I. Bennion, "Mirochannels in conventional single-mode fibers," Opt. Lett., vol. 31, no. 17, pp. 2559-2561, 2006. 